\title{
Involvement of EGFR in the promotion of malignant properties in multidrug resistant breast cancer cells
}

\author{
JIA-WEN XU ${ }^{1}$, QING-QUAN LI ${ }^{1}$, LI-LI TAO ${ }^{1}$, YUAN-YUAN CHENG ${ }^{1}$,

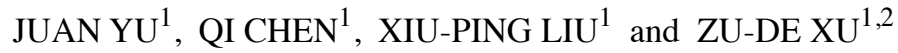 \\ ${ }^{1}$ Department of Pathology, Shanghai Medical College, ${ }^{2}$ Department of Pathology, \\ Huashan Hospital, Fudan University, Shanghai 200032, P.R. China
}

Received May 23, 2011; Accepted July 1, 2011

DOI: $10.3892 /$ ijo.2011.1143

\begin{abstract}
Multidrug resistance is the most predominant phenomenon leading to chemotherapy treatment failure in breast cancer patients. Despite many studies having suggested that overexpression of epidermal growth factor receptor (EGFR) is a potent predictor of malignancy in cancers, systematic research of EGFR in multidrug resistant (MDR) breast cancer cells is lacking. In order to clarify the role of EGFR in MDR breast cancer cells, MCF7/Adr expressing relatively higher EGFR, and its parental cell line MCF7 expressing relatively lower EGFR, were chosen for this study. Knockdown of EGFR by siRNA in MCF7/Adr cells showed that EGFR siRNA inhibits cell migration, invasion and proliferation in vitro; converse effects were observed in MCF7 cells transfected with pcDNA3.0-EGFR plasmid. Moreover, we found that EGFR upregulated migration and invasion via EMMPRIN, MMP2 and MMP9 in addition to promoting cell cycle passage via elevation of cyclin D1 and CDK4 in MDR breast cancer cells. Interestingly, MCF7/ Adr cells not expressing EGFR showed significant decrease of P-glycoprotein (P-gp) and ABCG2 expression levels, and became more sensitive to treatment of adriamycin (ADR) and paclitaxel (Taxol); the above results indicated that MDR of cancer cells is related to S-phase arrest. In conclusion, EGFR is an important factor enhancing the malignancy of MDR breast cancer cells, partially, inducing MDR. Anti-EGFR therapy may improve outcome in chemorefractory breast cancer patients.
\end{abstract}

\section{Introduction}

Breast cancer is the most prevalent health problem and is the second leading cause of death in women. Although chemotherapy treatment improved the survival rate in breast cancer for decades, more than $80 \%$ of patients who received chemo-

Correspondence to: Professor Zu-De Xu, Department of Pathology, Shanghai Medical College, Fudan University, Shanghai 200032, P.R. China

E-mail: zdxu@shmu.edu.cn

Key words: multidrug resistance, epidermal growth factor receptor, migration, invasion, cell cycle, breast cancer drugs will eventually develop multidrug resistance (MDR) after periods of treatment, leading to treatment failure. Therefore, new strategies to benefit MDR breast cancer patients is urgent required. Overexpression of ABC (ATP-binding-cassette) -transporters including $\mathrm{P}$-glycoprotein (P-gp), multidrug resistant protein 1-7 (MRP1-7) and ATP-binding cassette superfamily G member 2 (ABCG2) play important roles in effluxing different chemotherapy drugs out of tumor cells, which leads to the low concentration of drugs and treatment failure (1-3). Substantial evidence has shown inappropriate chemotherapy drugs use can enhance tumor malignancy (4). Moreover, our previous studies confirmed that MDR breast cancer cell line MCF7/Adr showed more aggressive malignant properties than its parental cell line MCF7 (5,6), but the underlying mechanism still needs further investigation.

EGFR is a member of HER tyrosine kinase family which can be activated by binding of specific ligands and leading to activation of downstream cellular signal transduction pathways. Numerous studies have shown that overexpression of EGFR is associated with poor prognosis in cancer (7-9). Interestingly, in MDR cells, EGFR appeared to be critically responsible for promoting invasion ability. Our previous study reported for the first time that treatment of MCF7/Adr cells with P-gp substrates could up-regulate the production of EGFR, extracellular matrix metalloproteinase inducer (EMMPRIN) and MMP2 and 9, consequently enhancing migratory/invasive abilities in vitro, moreover, the above pathway could be blocked by an EGFR inhibitor (6), suggesting EGFR may promote cell motility in MDR breast cancer cells, while the exact role of EGFR in MDR breast cancer still remains unclear.

Cell cycles are indispensable events that lead to cell division and duplication, not only in normal cells, but also in MDR cancer cells. The development of drug resistance phenotype could be accompanied by changes in morphological structure, proliferative potential and adhesion properties of cells as well as changes in expression of proteins involved in cell cycle control. Cell cycle-mediated drug resistance is best described as a relative insensitivity to a chemotherapeutic agent because of the position of the cells in the cell cycle. Different functions of EGFR in cell cycle regulation have been reported in various species, organs and cell lines $(10,11)$. Whether EGFR is involved in modulating cell proliferation and chemosensitivity in MDR breast cancer cells is yet to be clarified. 
In this study, we demonstrate that EGFR promotes MDR breast cancer cell migration/invasion by up-regulating EMMPRIN, MMP2 and 9 protein, and enhances cell proliferation via accelerating the cell cycle passage. We also evaluated the differential chemosensitivity of the G1 and S-synchronized breast cancer cells, and suggest a critical role for EGFR in impairing resistance in the $\mathrm{S}$ population. These studies suggest anti-EGFR treatment as a promising therapeutic strategy in MDR breast cancer.

\section{Materials and methods}

Cell culture. MDR breast cell line MCF7/Adr was cultured in RPMI-1640 (Gibco-BRL, Karlsruhe, Germany), and its parental cell line MCF7 was cultured in DMEM (high glucose) (Gibco-BRL) supplemented with $0.01 \mathrm{mg} / \mathrm{ml}$ bovine insulin (Sigma, St. Louis, MO, USA). All cell culture media contained $10 \%$ fetal bovine serum (FBS; PAA Laboratories, Linz, Austria), $100 \mathrm{U} / \mathrm{ml}$ penicillin and $100 \mu \mathrm{g} / \mathrm{ml}$ streptomycin. Cells were cultured at $37^{\circ} \mathrm{C}$ in a humidified atmosphere containing 5\% $\mathrm{CO}_{2}$. For consistent MDR-1 gene expression, MCF7/Adr cells were maintained in the presence of adriamycin (Sigma).

Immunofluorescence analysis. Cells seeded on 6-well chamber slides were fixed in $4 \%$ paraformaldehyde, permeabilized in $0.1 \%$ Triton X-100, and blocked in 5\% BSA. EGFR levels were detected using anti-EGFR (BD Biosciences, San Jose, USA), followed by incubation for $45 \mathrm{~min}$ with $\mathrm{Cy} 3$-conjugated antimouse secondary Ab (Amersham Biosciences, Uppsala, Sweden), finally the nuclei were stained with Hoechst 33258 (Sigma) for $5 \mathrm{~min}$ at room temperature. The labeled cells were analyzed by fluorescence microscopy (Zeiss, Oberkochen, Germany).

Inhibition of EGFR expression by RNA interference. Cells $\left(2 \times 10^{5}\right)$ were seeded in 6-well plates in triplicates and after an overnight incubation, the cells were transfected with various concentrations of siRNA using HiPerfect Reagent (Qiagen) as suggested by the manufacturer's instructions. The small interference RNA used to target EGFR mRNA sequence was synthesized by Qiagen.

Plasmid transfection. MCF7 cells were transfected with eukaryotic expression vector pcDNA3.0-EGFR (kindly provided by Professor Yosef Yarden) or pcDNA3.0 vector using Lipofectamine $^{\mathrm{TM}} 2000$ (Invitrogen, Carlsbad, CA, USA) following the manufacturer's protocol. For transfection, cells were grown to $80 \%$ confluence in a 6-well dish, $4 \mu \mathrm{g}$ of plasmid DNA and $10 \mu \mathrm{l}$ of Lipofectamine 2000 complexes were added in $2 \mathrm{ml}$ of Opti-MEM medium (Gibco-BRL). After $6 \mathrm{~h}$ incubation at $37^{\circ} \mathrm{C}$, culture medium was changed to usual complete medium and cells were cultured at $37^{\circ} \mathrm{C}$ for another $42 \mathrm{~h}$ until harvested.

Immunoblotting. Cells were collected and lysed in modified RIPA buffer [50 mM Tris (pH 7.8), $150 \mathrm{mM} \mathrm{NaCl,5}$ mM EDTA, $15 \mathrm{mM} \mathrm{MgCl} 2,1 \% \mathrm{NP}-40,0.5 \%$ sodium deoxycholate, $1 \mathrm{mM}$ DTT, and $20 \mathrm{mM} \mathrm{N}$-ethylmaleimide) containing 1 tablet $/ 50 \mathrm{ml}$ of Complete Protease Inhibitor Cocktail (Roche Molecular Biochemical, Indianapolis, IN, USA). Total cell lysate (50 $\mu \mathrm{g}$ protein) was resolved by standard sodium dodecyl sulphate polyacrylamide gel electrophoresis (SDS-PAGE) and electrophoretically transferred onto polyvinylidene fluoride (PVDF) membranes (Millipore, Bedford, MA, USA). After blocking in $5 \%$ non-fat milk for $1 \mathrm{~h}$ at room temperature, the membranes were first incubated overnight with primary antibodies against EGFR (BD Biosciences), $\beta$-actin (Sigma), EMMPRIN (Santa Cruz, USA), MMP2 (Cell Signaling Technology, USA), MMP9 (Abcam, USA), cyclin D1 (Cell Signaling Technology), CDK4 (Cell Signaling Technology), p21 (Cell Signaling Technology), p27 (Cell Signaling Technology), P-gp (Chemicon International, Temecula, CA), MRP1 (Santa Cruz) and ABCG2 (Santa Cruz) at $4^{\circ} \mathrm{C}$, respectively, and were then incubated for $1 \mathrm{~h}$ at $4^{\circ} \mathrm{C}$ with the appropriate HRP-conjugated secondary antibodies (Invitrogen). Detection of protein expression levels by enzymelinked chemiluminescence (ECL; Pierce, Rockford, USA) was performed according to the manufacturer's protocol.

Cell migration and invasion assays. For migration assay, Boyden dual chamber assay was performed using transwell chambers with $8-\mu \mathrm{m}$ pore size membranes (BD Biosciences). A total of $5 \times 10^{4}$ cells were suspended in serum-free media and added to the upper chamber, serum-positive media were used as chemoattractant in the lower chamber. After incubation for 48-72-h at $37^{\circ} \mathrm{C}$ with $5 \% \mathrm{CO}_{2}$, the media and cells remaining in the upper chamber were removed using a cotton swab. The insert was fixed in methanol and stained using hematoxylin and eosin. The number of invading cells was counted in five random per highpower fields and calculating the mean number of invading cells. All experiments were performed in triplicate.

The invasive capability of cells was assessed by using Boyden dual chamber assay as described previously with some modifications. The transwell chamber membranes were coated with $40 \mu 1$ of growth-factor-reduced Matrigel (BD Biosciences) for $4 \mathrm{~h}$ at $37^{\circ} \mathrm{C}$, and the assays were subsequently performed similar to those of the cell migration assays.

Cell proliferation assay. Cell proliferation was evaluated by a modified 3-(4,5-dimethylthiazol-2-yl)-2, 5-diphenyltetrazolium bromide (MTT, Roche Applied Science, USA) assay. The transfected and counterpart control cells were harvested by trypsinization and seeded at a concentration of $1.0 \times 10^{4}$ cells per well into 96-well plates. After 1-6 or 8-days culture at $37^{\circ} \mathrm{C}$ with $5 \% \mathrm{CO}_{2}$, the cells were washed twice with phosphate-buffered saline (PBS) and $100 \mu \mathrm{l}$ of culture medium supplemented with $20 \mu \mathrm{l}$ of $1 \mathrm{mg} / \mathrm{ml}$ of MTT was added to each well and incubated further for $4 \mathrm{~h}$. The medium containing MTT was then replaced with $150 \mu \mathrm{l}$ of dimethyl sulfoxide (DMSO) for $10 \mathrm{~min}$, and the spectrophotometric absorbancy was measured at $490 \mathrm{~nm}$ using ELISA multi-well spectrophotometer (Molecular Devices Corp., Sunnyvale, CA, USA). Each group contained 6-wells and was repeated three times.

DNA content analysis by flow cytometry was performed. In brief, cells were collected by trypsinization, pelleted by centrifugation and washed twice with PBS at $48 \mathrm{~h}$ after transfection, and then were resuspended and fixed overnight at $-20^{\circ} \mathrm{C}$ with $70 \%$ ethanol pre-cooled. The relative data of DNA content was measured and analysized by using a fluorescent-activated cell sorter (FACS) Canto flow cytometer. The proportions of cells in $\mathrm{G} 1, \mathrm{~S}$ and $\mathrm{G} 2 / \mathrm{M}$ and the apototisis rates were calculated using FACS DiVa software package (BD Biosciences). 

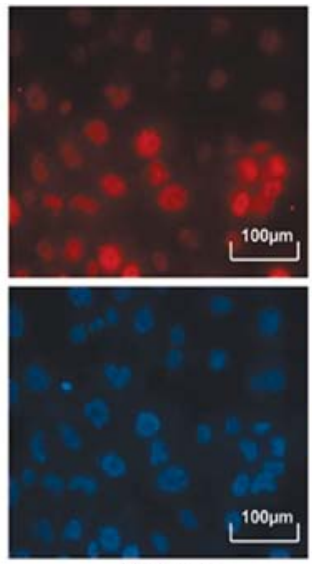

MCF7/Adr
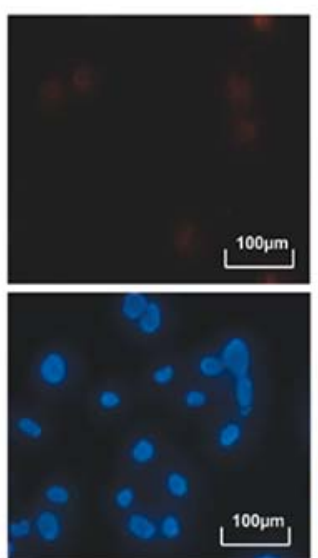

MCF7
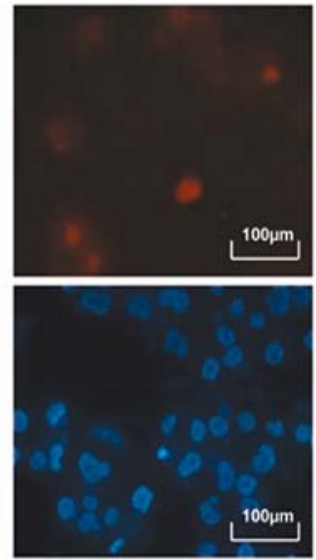

MCF7/Adr-EGFR siRNA
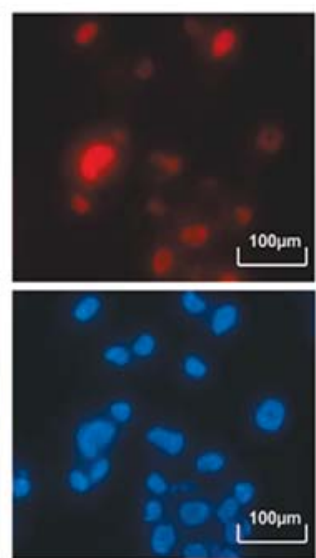

MCF7/pcDNA3.0-EGFR
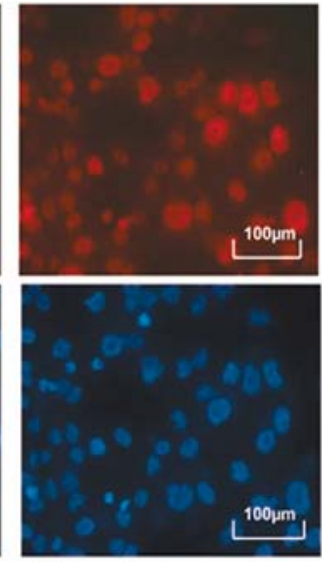

MCF7/Adr Negative control
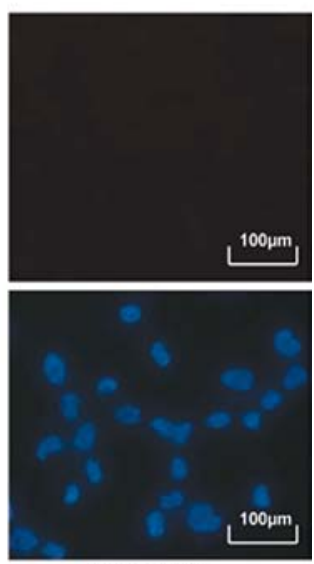

MCF7/vector

B
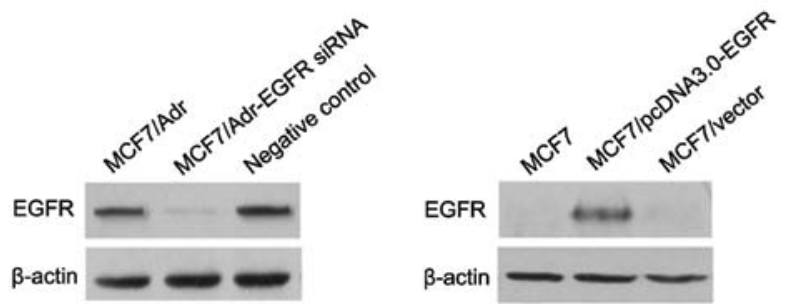

Figure 1. Production of EGFR protein in MCF7/Adr, MCF7 cells were down- or upregulated after transfecting EGFR siRNA and pcDNA3.0-EGFR plasmid, respectively. (A) Transfection efficiencies identified by immunofluorescence, the nuclei were stained with Hoechst 33258 . All fields are representative of multiple fields observed in at least three independent experiments. (B) EGFR protein assessed by Western blot analysis. $\beta$-actin served as control for sample loading.

Cell cycle synchronization. Cells arrested in G1-phase were achieved by $48 \mathrm{~h}$ incubation in serum-free medium. Double Thymidine ( $2 \mathrm{mM}, 16 \mathrm{~h}$ ) Block method is used for synchronizing cells in S-phase. Cell cycle distributions were determined using flow cytometry as described.

Drug sensitivity assay. Multidrug chemosensitivity of transfected cells and their corresponding control cells were plated in 96-well plates at a density of $10^{4}$ cells/well and further incubated for $24 \mathrm{~h}$. The medium was then removed and replaced with fresh medium containing adriamycin (ADR) and paclitaxe 1 (Taxol) (Sigma), respectively, with different PPC (plasma peak concentrations, OPPC, 0.1 PPC, 1.0 PPC and 10.0 PPC) for another $48 \mathrm{~h}$. The assays were subsequently performed by MTT. The $\mathrm{IC}_{50}$ value was determined by the dose of drug that caused $50 \%$ cell viability. (1PPC of Adr: $0.4 \mu \mathrm{g} / \mathrm{ml}, 1 \mathrm{PPC}$ of Taxol: $4.5 \mu \mathrm{g} / \mathrm{ml})$.
Statistical analysis. Statistics were calculated by SPSS software. All experiments were repeated at least three times and the the results are presented with mean \pm standard errors (SEM). The differences were analysized by using the ANOVA and Student's $\mathrm{t}$-test and the statistical significance was determined at $\mathrm{p}<0.05$ (two-tailed).

\section{Results}

EGFR-siRNA and pcDNA3.0-EGFR plasmid effectively downor upregulated EGFR expression, respectively. To assess the role of EGFR in MDR breast cancer cells, EGFR-siRNA was transfected in MCF7/Adr cells while pcDNA3.0-EGFR plasmid was transfected in MCF7 cells as a control test. As shown in Fig. 1A, the fluorescence intensity which represented EGFR expression level in EGFR-siRNA and pcDNA3 .0-EGFR- transfectants displayed a significant reduction or promotion when 
A a
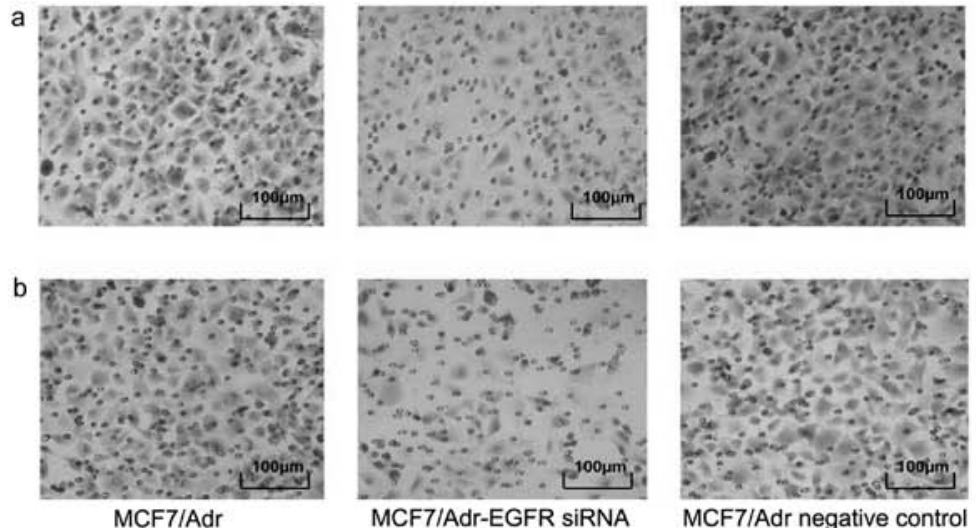

MCF7/Adr

MCF7/Adr-EGFR siRNA

MCF7/Adr negative control
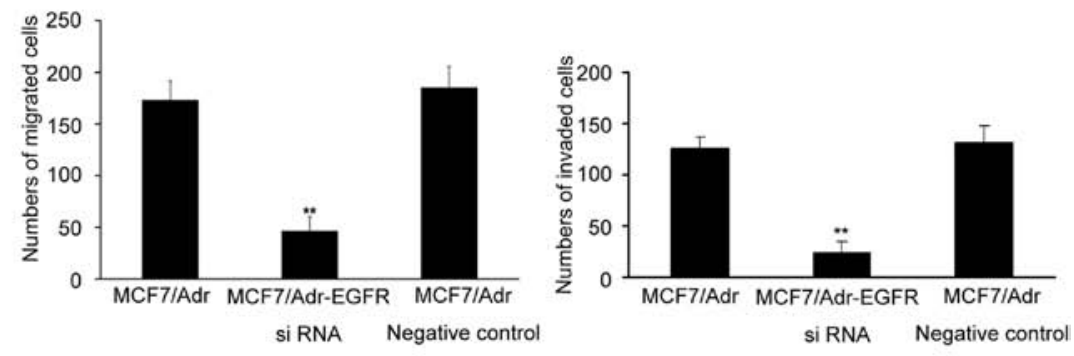

B
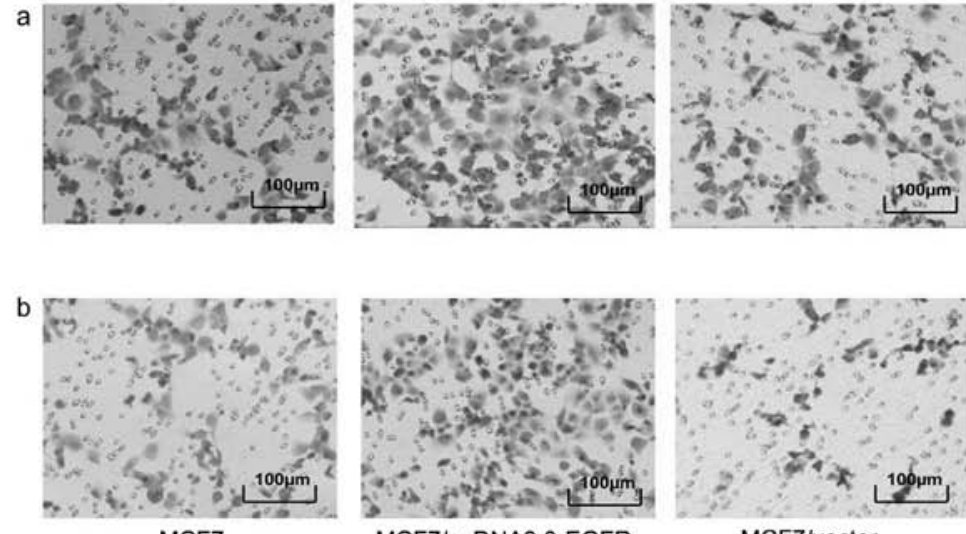

MCF7/pCDNA3.0-EGFR

MCF7/vector
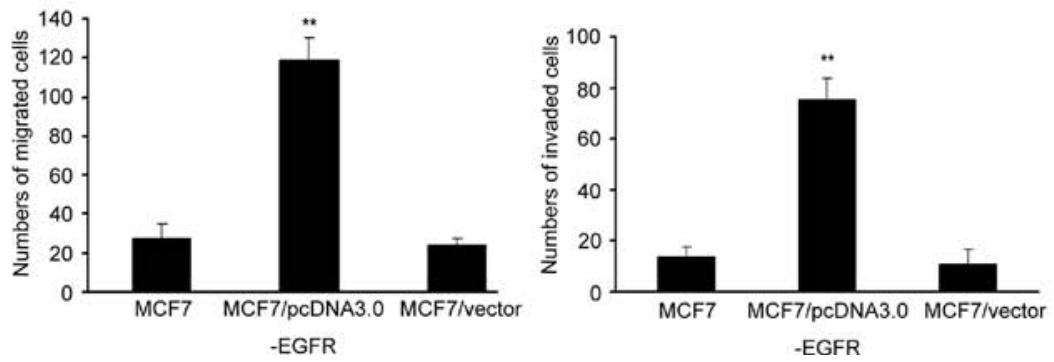

Figure 2. Effects of EGFR on migration and invasion in MCF7/Adr and MCF7 cells. (A) EGFR-knockdown and control groups; (B) EGFR-plasmid transfection and control groups. Migration (a) and invasion (b) were evaluated by using transwell chamber assays as described before. The bar graphs show the number of cells migrated or invaded in five fields for each experimental group as an average, corresponding to the upper figures. Statistical analysis was carried out with Dunnett's-test. ${ }^{* *} \mathrm{p}<0.05$ vs control cells.

compared with their negative controls. In addition, Western blot analysis showed the responsive changes in corresponding transfectants (Fig. 1B).

EGFR enhances in vitro cell migration and invasion via upregulating EMMPRIN, MMP2 and MMP9 in MDR breast cancer cells. In order to verify whether EGFR modulate the migration and invasion ability, transwell assay without or with Matrigel was performed. EGFR-siRNA transfected MCF7/Adr cells showed significantly decreased migration and invasion ability compares to the control groups (Fig. 2). EMMPRIN, MMP2 and MMP9 proteins have been reported to be important factors 

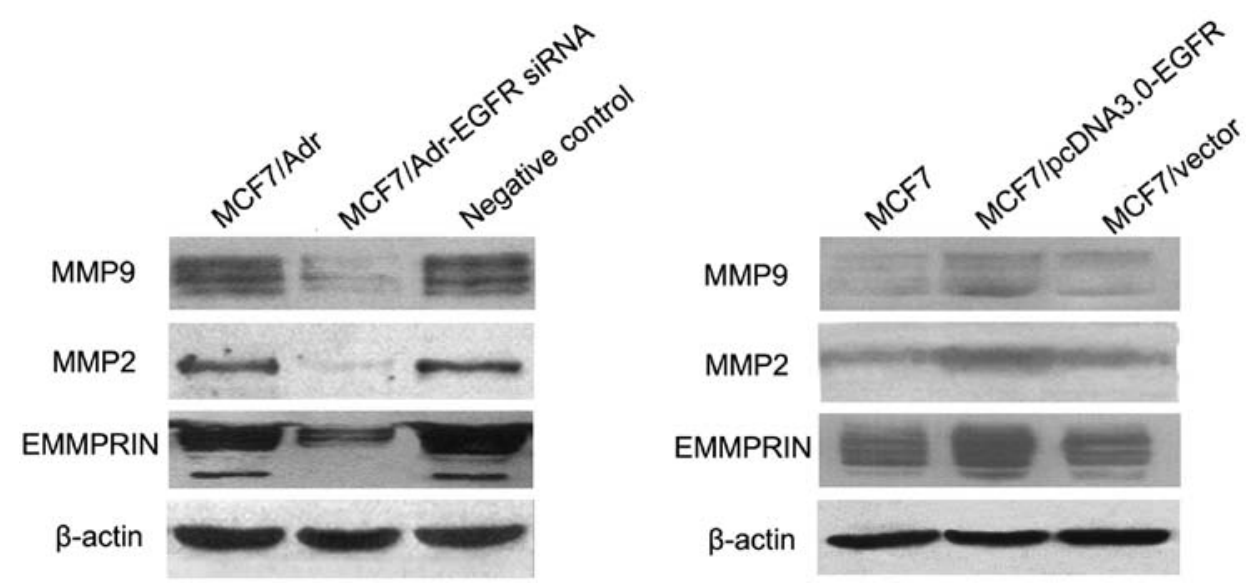

Figure 3. Effects of EGFR on expression of EMMPRIN, MMP2 and MMP9 in MCF7/Adr and MCF7 cells. EMMPRIN, MMP2 and MMP9 protein levels assessed by Western blot analysis. $\beta$-actin served as control for sample loading.

Table I. $\mathrm{IC}_{50}$ of chemotherapeutic agents in EGFR transfectants.

\begin{tabular}{lcccc}
\hline Drugs & MCF7/Adr & MCF7/Adr-EGFR siRNA & MCF7/vector & MCF7/EGFR \\
\hline ADR & $17.5452 \pm 3.1202$ & $7.8393 \pm 0.7596^{\mathrm{a}}$ & $2.6152 \pm 1.1085$ & $4.2623 \pm 0.4949^{\mathrm{a}}$ \\
Taxol & $26.7799 \pm 1.4857$ & $7.6273 \pm 1.6012^{\mathrm{a}}$ & $3.5934 \pm 1.1031$ & $16.146 \pm 2.9152^{\mathrm{a}}$ \\
\hline
\end{tabular}

$\mathrm{ADR}$, adriamycin; Taxol, paclitaxel; $\mathrm{IC}_{50}$ values were expressed in $\mu \mathrm{g} / \mathrm{ml}$ and were evaluated as reported in Materials and methods. Standard deviations for all of the experiments performed in triplicate were $<5 \%$. ${ }^{\mathrm{a}} \mathrm{p}<0.05$ versus control cells.

involved in promoting cancer cell migration and invasion. As shown in Fig. 3, EMMPRIN, MMP2 and MMP9 levels were significantly lower in EGFR-silenced MCF7/Adr cells than in control groups. As a converse test, EGFR-transfected MCF7 cells showed the opposite results. These data confirmed that EGFR promotes in vitro cell migration and invasion not only in sensitive MCF7 cells, but also in MDR breast cancer cells.

EGFR enhances in vitro cell proliferation via upregulating cyclin D1, CDK4 and downregulating p21, p27 in MDR breast cancer cells. To determine the effect of EGFR on the tumorigenic properties of MDR breast cancer cells, we assessed the growth capacity of EGFR-silenced MCF7/Adr cells using MTT assay. As shown in Fig. 4A, knockdown EGFR expression in MCF7/Adr cells efficiently suppressed cell growth, while elevated EGFR expression in MCF7 cells led to the opposite effect when compared with negative controls.

In order to confirm the observation, we examined the cell cycle distribution of EGFR-silenced MDR cells by flow cytometry. As shown in Fig. 4B and C, depletion of EGFR in MCF7/Adr cells override S-phase arrest while ectopic EGFR expression lead to S-phase arrest in MCF7 cells, without affecting the distribution of $\mathrm{G} 2 / \mathrm{M}$-phase and rate of apoptosis.

To investigate the preliminary mechanisms involved in the regulation of cell proliferation by EGFR in MDR breast cells, the expression of cyclin D1, CDK4, p21 and p27 in the EGFRsiRNA transfected MCF7/Adr cells were analyzed by Western blot and compared with that of control cells (Fig. 4D). Our data revealed remarkable downregulation in the expression of cell cycle-related proteins cyclin D1 and CDK4, as well as elevation in the expression of p21 and p27. Whereas, pcDNA3.0-EGFRtransfected MCF7 showed the opposite effect when compared with negative controls (Fig. 4D).

Effect of EGFR in modulating chemosensitivity in MDR breast cancer cells. MDR is best described as a relatively insensitive to a chemotherapeutic agent, in order to verify the role of EGFR in regulating MDR properties, chemosensitivity to P-gp-related drugs ADR and Taxol was assessed by MTT assay in this study, as reported in Table I, The EGFR-silenced MCF7/Adr cells showed decreased resistance to ADM and Taxol compared with the control groups. However, all these effects were remarkably reversed upon EGFR upregulation in MCF7 cells.

To study the possible molecular mechanisms involved in the MDR of breast cancers, the expression level of 3 classical MDR molecules, P-gp, MRP1 and ABCG2, was examined by Western blot analyses. Interestingly, we found P-gp and ABCG2 levels were obviously decreased after the knockdown of EGFR in MCF7/Adr cells paralleled with the control groups. Whereas, ectopic EGFR expression in MCF7 cells led to the opposite effects (Fig. 5). However, the expression of MRP1 remained unchanged (Fig. 5). These results indicated that EGFR had a crucial role in increasing MDR in breast cancer cells by enhancing P-gp and ABCG2 expression.

The MDR phenotype is cell cycle-dependent in breast cancer cells. The abnormal regulation of the cell cycle is known as one of the characteristic features of the malignant cells. In our 

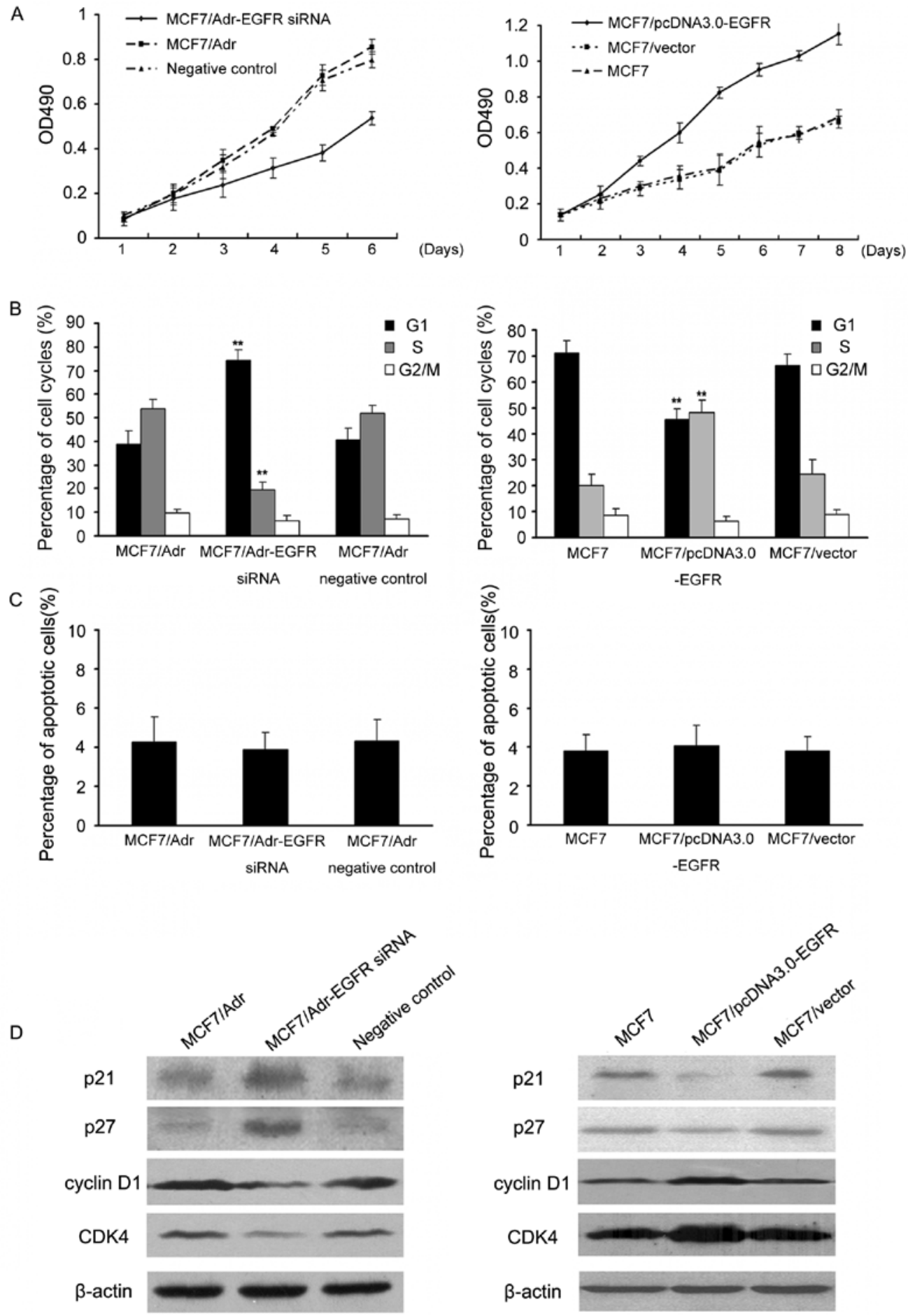

Figure 4. Effects of EGFR on the cell growth in MCF7/Adr and MCF7 cells. MCF7/Adr and MCF7 cells were transfected with EGFR siRNA and pcDNA3.0-EGFR plasmid, respectively. (A) Cell growth curve measured by MTT assay. Each point indicates the mean of spectrometric absorbance \pm SEM of three independent experiments. (B) Cells cycle analyzed by flow cytometry. Each bar indicates the distribution of cells cycle. (C) Cell apoptotic ratio analyzed by flow cytometry. Each bar indicates the mean of cell apoptosis \pm SEM of three independent experiments. ${ }^{* *} \mathrm{p}<0.05$ vs. control cells. (D) Cyclin D1, CDK4, p21 and p27 protein assessed by Western blot analysis. $\beta$-actin served as control for sample loading.

previous data, we found $>50 \%$ of MCF7/Adr cells were arrested at S-phase, whereas most MCF7 cells were in G1-phase under normal culture conditions. To determine if the acquisition of the MDR phenotype was attributed to the S-phase arrest, MCF7 cells were synchronized in the G1-phase or in the S-phase. It was observed that with double thymidine treatment, the $\mathrm{S}$-arrested cells were significantly more resistant to ADR and
Taxol in comparison with the cells arrested at G1-phase or the unsynchronized controls (Table II). P-gp, MRP1 and ABCG2 were also examined in the cells synchronized at different phases. The expressions of both P-gp and ABCG2 in the S-arrested cells were remarkably higher than those in the G1-arrested cells and the unsynchronized cells (Fig. 6). However, the expression of MRP1 still remained unchanged upon cell cycle synchroniza- 


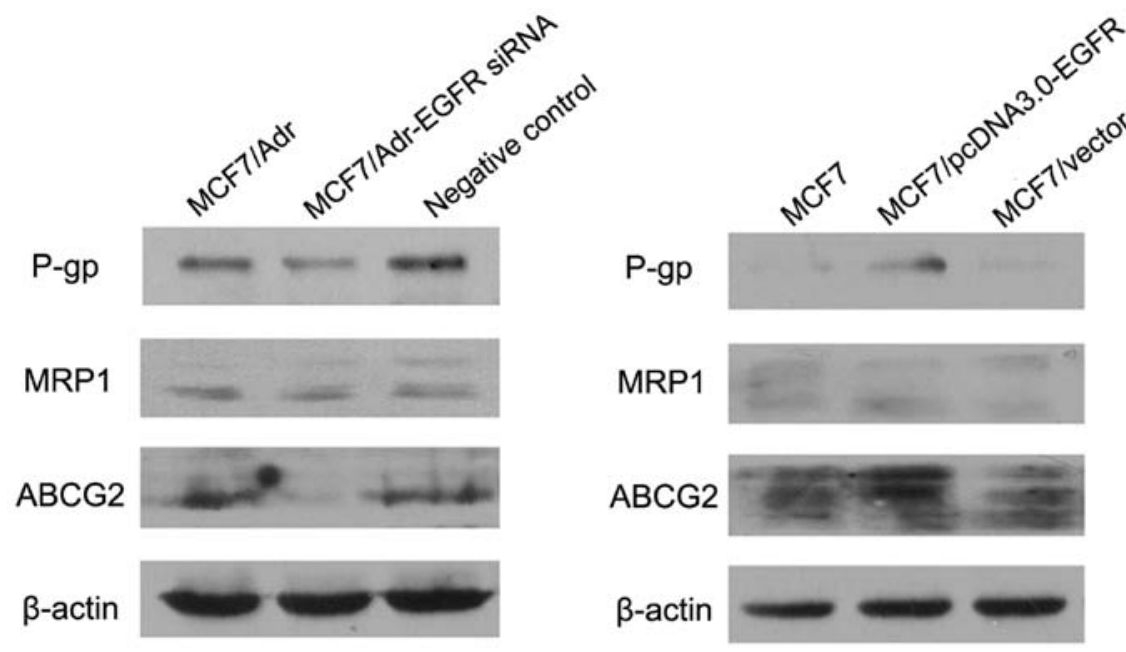

Figure 5. Effects of EGFR on the MDR modulation in MCF7/Adr and MCF7 cells. P-gp, MRP1 and ABCG2 protein assessed by Western blot analysis. $\beta$-actin served as control for sample loading.

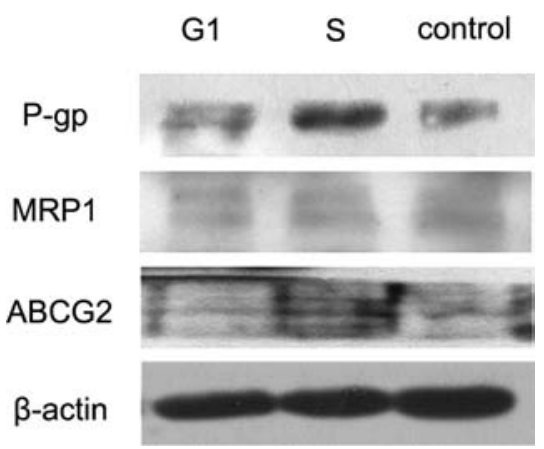

Figure 6. Upregulation of P-gp and ABCG2 is cell cycle-dependent in breast cancer cells. After MCF7 cells were synchronized at G1 or S-phase, the expression level of P-gp, ABCG2 and MRP1 was determined by Western blot analysis. $\beta$-actin served as control for sample loading.

Table II. $\mathrm{IC}_{50}$ of chemotherapeutic agents in different cell cycle phases of MCF7 cells.

\begin{tabular}{lccc}
\hline Drugs & G1 & $\mathrm{S}$ & Unsynchronized \\
\hline ADR & $2.4842 \pm 0.7520$ & $8.5772 \pm 2.0353^{\mathrm{a}}$ & $2.6152 \pm 1.1086$ \\
Taxol & $3.0494 \pm 0.5890$ & $7.4993 \pm 1.1682^{\mathrm{a}}$ & $3.5934 \pm 1.1031$ \\
\hline
\end{tabular}

$\mathrm{IC}_{50}$ values were expressed in $\mu \mathrm{g} / \mathrm{ml}$ and were evaluated as reported in Materials and methods. Standard deviations for all of the experiments performed in triplicate were $<5 \%$. ${ }^{\mathrm{a}} \mathrm{p}<0.05$ versus control cells.

tion (Fig. 6). Taken together, we supposed that EGFR is able to induce MDR by accelerating G1/S transition, whch enabling higher P-gp and ABCG2 produced.

\section{Discussion}

Breast cancer is one of the most common neoplasms in women worldwide, and is also the principal cause of tumor-related death in women (12). Although over the past several decades, strategies for treatment of breast cancer has undergone great developments, no effective cure for breast cancer exists. Chemotherapy is a systemic treatment eliminated effectively cancer cells, however, American Cancer Society has reported that $>90 \%$ of cancer patients do not fully respond to chemodrugs and multidrug resistance in the courses of treatment, finally results in treatment failure (i.e., cancer recurrence), so the effective handling of the MDR reversal constitutes the most urgent problem that calls for immediate solution.

EGFR is an important tyrosine kinase receptor linked with human cancer, locating on chromosomes 7p11.2 and encoding a $170-\mathrm{kDa}$ transmembrane protein, involved in the ErbB signaling pathway (13). Remarkable association between EGFR expression and poor prognosis has been reported in breast cancer patients treated with different chemotherapy strategies but not in non-chemotherapy group (14), indicating that EGFR expression is probably connected to chemo-drug application. Correspondingly, we previously reported that EGFR expression was at higher level in MCF7/Adr breast cancer cells than in the sensitive MCF7 cells (9), however, the role EGFR plays in MDR breast cancer cells is still not fully elucidated. Therefore, in this study, we knocked down EGFR expression in MCF7/Adr cells by siRNA transfection, and were surprised to find the migrative/invasive abilities of transfected cells decreased significantly, whereas elevated EGFR expression in MCF7 cells showed the opposite effect. EMMPRIN (CD147 or basigin) is a transmembrane protein closely related to promoting migration/invasion in many types of solid tumors, including breast cancer, which can efficiently stimulate stromal cells and even cancer cells themselves to secrete matrix metalloproteases (MMPs) $(15,16)$. MMP2 and MMP9 are the most correlative MMPs in enhancing breast cancer metastasis. We have reported before that EMMPRIN effectively promoted tumor invasion in vitro as well as increasing resistance to P-gp substrate drugs in MCF7/Adr cells, however, whether EGFR participated in the regulation of EMMPRIN in MCF7/Adr cells is still obscure. In this study, we tested the expression of EMMPRIN, MMP2 and MMP9 after knockdown of EGFR expression in MCF7/adr cells, and the results showed all three migration-related proteins were 
extremely downregulated. As a reverse authentication, atopic EGFR expression in MCF7 cells was the opposite. In accordance with our research, Menashi et al found amphiregulin (a kind of ligand for EGFR) evidently induced EMMPRIN and MMPs via EGFR tyrosine kinase activation in transformed human breast epithelial cells (17). All observations suggest that EGFR plays a crucial part in enhancing EMMPRIN and MMPs expression to establish favorable conditions for cell migration/invasion not only in generalized breast cancer cells, but also in MDR breast cancer cells, indicating MDR patients with EGFR overexpression might benefit from novel therapies targeting EGFR.

Cancer cell proliferation has been shown to be one of the most significant predictors of survival in breast cancer patients. Although research has linked EGFR overexpression with proliferation in certain types of cells in vivo or in vitro, whether the proliferation of MDR breast cancer cells is regulated by EGFR was not clear. In the current study, reduction of EGFR expression in MCF7/Adr cells significantly inhibited the cell viability without affecting the apoptosis rate, while up-regulation of EGFR expression efficiently promoted cell proliferation in MCF7 cells. The marked impact of EGFR on cell proliferation may be related to the wide variety of signaling factors to which EGFR binds in a cell-type specific manner, such as EGF, TGF- $\alpha$, amphiregulin, leading to activation of the downstream signaling pathway that resulted in accelerating cell growth (18). It is well known that cell cycle regulation is a prominent factor in normal cell proliferation, while defect in cell cycle control can cause unchecked growth in cancer cells (19). Our results showed G1-phase arrest in EGFR-silenced MCF7/Adr cells, accompanied by a significant reduction in S-phase, illustrating the G1/S transition meets with obstruction, however, the percentage of G2/Mphase was not influenced. To investigate the exact mechanism of EGFR affecting cell cycle in MDR breast cancer cells, cell cycle regulator related protein levels were assessed in EGFR-knockdown MCF7/Adr cells. The results showed that down-regulation of EGFR leading to inhibition of expression of cyclin D1 and CDK4, as well as increasing p21 and p27 protein levels. These results are consistent with Grillo et al (20), and confirmed that cyclin D1/CDK4 complex is a key cell cycle regulator in increasing passage through $\mathrm{G} 1 / \mathrm{S}$ boundary in MDR breast cancer cells, and their levels can be modulated by EGFR expression. Moreover, p21 and p27 were recognized as two universal inhibitors of cyclin-dependent kinase (CDK), and negative regulators in the G1/S transition. Although the p21 and p27 may also act as oncogenes, down-regulation or loss of $\mathrm{p} 21$ expression were also found in a series of human solid tumors, and resulted in uncontrollable cell proliferation (21-23). In this study, we considered that p21 and p27 are involved in inhibiting cyclin-CDK activity, and consequently resulted in G1-phase arrest. Although cell cycle arrest by specific abrogation of EGFR was reported in a series of cancer cells (24), this is the first demonstration that EGFR can modulate cell cycle progress in MDR breast cancer cells, which pave the way for application of anti-EGFR treatment in chemorefractory breast cancer patients.

MDR is mediated by complex mechanisms which are severe challenge for both basic and clinical researchers. The most investigated mechanism with known clinical significance is activation of the $\mathrm{ABC}$ transporter family that can act as cell membrane pumps effluxing different chemical substances from the cells (25). We report that P-gp, MRP1 and ABCG2 expression in EGFR knockdown MCF7/Adr cells, and find P-gp and ABCG2 expression decreased sharply after siRNA transfection. On the contrary, both of them were elevated in EGFR-transfected MCF7 cells, while the MRP1 showed no significant change in the experiments. Moreover, EGFR-silenced MCF7/Adr cells showed greater sensitivity towards ADR and Taxol, which were both recognized as P-gp substrates and used widely in breast cancer chemotherapy. A consistent finding was reported in HEK293 cells that EGFR tyrosine inhibitor AG1478 is able to inhibit the function of P-gp and with a more pronounced effect on ABCG2 (26). It is becoming increasingly apparent that the cell cycle plays a critical role in regulating chemosensitivity of cancer cells. Cell cycle-mediated drug resistance is best described as a relative insensitivity to a chemotherapeutic agent because of the position of the cells in the cell cycle. The degree of S-phase arrest was positively associated with the levels of MDR and proliferative rate in breast cancer cells. It has been suggested that tumor with a more rapid growth rate might be more sensitive to chemotherapy (27). We observed enhanced chemoresistance in breast cancer cells synchronized at S-phase, whereas a dramatic increased sensitivity to chemotherapeutic agents was detected in the cells at G1-phase. These results implied that the most likely factor that determines the susceptibility of breast cancer cells to chemotherapeutic agents, and thus the tumor cell growth, lies in the cell cycle stages. In our study, the expression of cyclin D1 and CDK4 was found to be downregulated in MDR cells transfected with EGFR-siRNA. Taken together, EGFR might facilitate cell chemorefractory activity by accelerating the expression of cyclin D1 and CDK4.

Our study demonstrated the role of EGFR in modulating migration/invasion, proliferation and chemo-drug resistance in MDR breast cancer cells. Since a number of EGFR inhibitors have been developed that can restore chemosensitivity and arrest tumor growth, a better understanding of the molecular mechanisms of action of EGFR in this study may contribute to the development of further strategies for integration of EGFR inhibitors with chemotherapy or radiation to potentiate their anticancer activity.

\section{Acknowledgements}

This work was supported by grants from the National Nature Science Foundation of China (No.30872971) and Research Fundation for the Doctoral Program of Higher Education of China (No. 200802460042). We thank members of our laboratory for helpful discussions.

\section{References}

1. Shustik C, Dalton W and Gros P: P-glycoprotein mediated multidrug resistance in tumor cells: biochemistry clinical relevance and modulation. Mol Aspects Med 16: 1-78, 1995.

2. Twentyman PR and Versant voort CH: Experimental modulation of MRP (multidrug resistance-associated protein)-mediated resistance. Eur J Cancer 32: 1002-1009, 1996.

3. Chen YN, Mickley LA, Schwartz AM, Acton EM, Hwang JL and Fojo AT: Characterization of adriamycin-resistant human breast cancer cells which display overexpression of a novel resistancerelated membrane protein. J Biol Chem 265: 10073-10080, 1990 . 
4. Choi S, Kobayashi M, Wang J, Habelhah H, Okada F, Hamada J, Moriuchi T, Totsuka Y and Hosokawa M: Activated leukocyte cell adhesion molecule (ALCAM) and annexin II are involved in the metastatic progression of tumor cells after chemotherapy with Adriamycin. Clin Exp Metastasis 18: 45-50, 2000.

5. Li QQ, Wang WJ, Xu JD, Cao XX, Chen Q, Yang JM and Xu ZD: Involvement of CD147 in regulation of multidrug resistance to P-gp substrate drugs and in vitro invasion in breast cancer cells. Cancer Sci 98: 1064-1069, 2007.

6. Li QQ, Wang WJ, Xu JD, Cao XX, Chen Q, Yang JM and Xu ZD: Up-regulation of CD147 and matrix metalloproteinase-2, -9 induced by P-glycoprotein substrates in multidrug resistant breast cancer cells. Cancer Sci 98: 1767-1774, 2007.

7. De Castro-Carpeño J, Belda-Iniesta C, Casado Sáenz E, Hernández Agudo E, Feliu Batlle J and González Barón M: EGFR and colon cancer: a clinical view. Clin Transl Oncol 10: 6-13, 2008

8. Gazdar AF: Epidermal growth factor receptor inhibition in lung cancer: the evolving role of individualized therapy. Cancer Metastasis Rev 29: 37-48, 2010.

9. Araújo AP, Catarino R, Ribeiro R, Pereira D, Pinto D and Medeiros R: Epidermal growth factor genetic variation associated with advanced cervical cancer in younger women. Am J Clin Oncol: Feb 22, 2011 (Epub ahead of print).

10. Santiskulvong C, Sinnett-Smith J and Rozengurt E: EGF receptor function is required in late $\mathrm{G}(1)$ for cell cycle progression induced by bombesin and bradykinin. Am J Physiol Cell Physiol 281: 886-898, 2001.

11. Baker NE and Yu SY: The EGF receptor defines domains of cell cycle progression and survival to regulate cell number in the developing Drosophila eye. Cell 104: 699-708, 2001.

12. Bray F, McCarron P and Parkin DM: The changing global patterns of female breast cancer incidence and mortality. Breast Cancer Res 6: 229-239, 2004

13. Hynes NE and Lane HA: ERBB receptors and cancer: the complexity of targeted inhibitors. Nat Rev Cancer 5: 341-354, 2005 .

14. Rimawi MF, Shetty PB, Weiss HL, Schiff R, Osborne CK, Chamness GC and Elledge RM: Epidermal growth factor receptor expression in breast cancer association with biologic phenotype and clinical outcomes. Cancer 116: 1234-1242, 2010.

15. Egeblad M and Werb Z: New functions for the matrix metalloproteinases in cancer progression. Nat Rev Cancer 2: 161-174, 2002.

16. Sternlicht MD and Werb Z: How matrix metalloproteinases regulate cell behavior. Annu Rev Cell Dev Biol 17: 463-516, 2001.
17. Menashi S, Serova M, Ma L, Vignot S, Mourah S and Calvo F: Regulation of extracellular matrix metalloproteinase inducer and matrix metalloproteinase expression by amphiregulin in transformed human breast epithelial cells. Cancer Res 63: 7575-7580, 2003.

18. Harris RC, Chung E and Coffey RJ: EGF receptor ligands. Exp Cell Res 284: 2-13, 2003.

19. Srivastava SK and Singh S: Cell cycle arrest, apoptosis induction and inhibition of nuclear factor kappa $B$ activation in antiproliferation activity of benzyl isothiocyanate against human pancreatic cancer cells. Carcinogenesis 25: 1701-1709, 2004.

20. Grillo M, Bott MJ, Khandke N, McGinnis JP, Miranda M, Meyyappan M, Rosfjord EC and Rabindran SK: Validation of cyclin D1/CDK4 as an anticancer drug target in MCF-7 breast cancer cells: Effect of regulated overexpression of cyclin D1 and siRNA-mediated inhibition of endogenous cyclin D1 and CDK4 expression. Breast Cancer Res Treat 95: 185-194, 2006.

21. Shah MA, Kortmansky J, Motwani M, Drobnjak M, Gonen M, Yi S, Weyerbacher A, Cordon-Cardo C, Lefkowitz R, Brenner B, O'Reilly E, Saltz L, Tong W, Kelsen DP and Schwartz GK: A phase I clinical trial of the sequential combination of irinotecan followed by flavopiridol. Clin Cancer Res 11: 3836-3845, 2005.

22. Rau B, Sturm I, Lage H, Berger S, Schneider U, Hauptmann S, Wust P, Riess H, Schlag PM, Dörken B and Daniel PT: Dynamic expression profile of p21WAF1/CIP1 and Ki-67 predicts survival in rectal carcinoma treated with preoperative radiochemotherapy. J Clin Oncol 21: 3391-3401, 2003.

23. Goan YG,Hsu HK, Chang HC, Chou YP, Chiang KH and Cheng JT: Deregulated p21(WAF1) overexpression impacts survival of surgically resected esophageal squamous cell carcinoma patients. Ann Thorac Surg 80: 1007-1016, 2005.

24. Lui VW and Grandis JR: EGFR-mediated cell cycle regulation. Anticancer Res 22: 1-11, 2002.

25. Stavrovskaya AA: Cellular mechanisms of multidrug resistance of tumor cells. Biochemistry 65: 95-106, 2000.

26. Shi Z, Tiwari AK, Shukla S, Robey RW, Kim IW, Parmar S, Bates SE, Si QS, Goldblatt CS, Abraham I, Fu LW, Ambudkar SV and Chen ZS: Inhibiting the function of ABCB1 and ABCG2 by The EGFR tyrosine kinase inhibitor AG1478. Biochem Biophys Res Commun 77: 781-793, 2009.

27. Hine KR and Dykes PW: Prospective randomised trial of early cytotoxic therapy for recurrent colorectal carcinoma detected by serum CEA. Gut 25: 682-688, 1984 